\title{
Uncertainties of ISO 3382-3 sound pressure level quantities
}

\author{
Moritz Schneider*, Jan Selzer*, Jörg Rissler, Andrea Wolff, and Florian Schelle \\ Institute for Occupational Safety and Health of the German Social Accident Insurance (IFA), 53757 Sankt Augustin, Germany
}

Received 9 September 2020, Accepted 30 May 2021

\begin{abstract}
The ISO 3382-3 standard uses the measurable sound pressure based parameters $D_{2, \mathrm{~S}}$ and $L_{p, \mathrm{~A}, \mathrm{~S}, 4 \mathrm{~m}}$ to describe the acoustic properties of open-plan offices. As yet however, no treatment of the measurement uncertainty of these parameters according to the Guide to the expression of Uncertainty in Measurement (GUM) is to be found in the peer-reviewed literature. This technical note therefore describes how the measurement uncertainty can be declared according to GUM. The mathematical framework presented here can be used and expanded by other laboratories to derive their own uncertainty estimates. It is also applied in this document to 44 measurements yielding combined uncertainties for $D_{2, \mathrm{~S}}$ of $0.55 \mathrm{~dB} \leq \sigma_{c, D_{2 S}} \leq 0.67 \mathrm{~dB}$ and for $L_{p, \mathrm{~A}, \mathrm{~S}, 4 \mathrm{~m}}$ of $0.19 \mathrm{~dB} \leq \sigma_{c, L_{p, \mathrm{~A}, \mathrm{~S}, 4 \mathrm{~m}}} \leq 0.83 \mathrm{~dB}$. The implications of this result are discussed with regard to limit values in technical regulations.
\end{abstract}

Keywords: Office, Uncertainty, Classification, ISO 3382-3, GUM

\section{Introduction}

Work in open-plan offices is often associated with complaints of poor privacy and overall dissatisfaction with the acoustic situation (e.g. [1, 2]). Higher sick leave rates are also reported for open-plan offices and flex-offices than for celloffices [3]. Furthermore, speech has been shown to impair performance (e.g. [4]). Surveys and questionnaires are appropriate means of identifying the load or stress upon the exposed office workers. However, the planning and design of technical measures to improve the acoustic situation in an open-plan office requires room acoustic descriptors. Since the reverberation time is not sufficient to describe the room acoustics of an open-plan office, ISO 3382-3 [5] defines a set of six further acoustic parameters, of which the spatial decay rate of speech $D_{2, \mathrm{~S}}$ and the A-weighted sound pressure level of speech at a distance of $4 \mathrm{~m} L_{p, \mathrm{~A}, \mathrm{~S}, 4 \mathrm{~m}}$ are relevant for this elaboration.

Unfortunately, due to the new method at the time of publication, ISO 3382-3 contains no information on the uncertainty of measurement, even though it obviously exists [6-9]. This presents an obstacle to the assessment of technical protective measures where their effect is equal or lower in magnitude to the measurement uncertainty. The same is true where measurement results from different laboratories for which no measurement uncertainty is stated are compared. Thirdly, the measurement uncertainty is crucial in comparisons of measurement results with limit values and for the determination of limit values themselves.
The Guide to the expression of Uncertainty in Measurement (GUM) [10] provides a general, mathematical framework for describing the effect of different uncertainty components. However, to our knowledge no peer-reviewed publication currently exists presenting results for the measurement uncertainty of $D_{2, \mathrm{~S}}$ and $L_{p . \mathrm{A} . \mathrm{S} .4 \mathrm{~m}}$ with reference to the GUM. Few uncertainty components for ISO 3382-3 measurements have been addressed in the literature, so far. Given the same office conditions, Yadav et al. [6] measured two different paths and a single path, with two different speaker positions in each case. From these two measurements, a "within measurement" standard deviation of $0.44 \mathrm{~dB} \leq \sigma_{w} \leq 0.61 \mathrm{~dB}$ for $D_{2, \mathrm{~S}}$ and of between $1.04 \mathrm{~dB}$ and $1.61 \mathrm{~dB}$ for $L_{p, \mathrm{~A}, \mathrm{~S}, 4 \mathrm{~m}}$ is derived. The same authors refer to Haapakangas et al. [7], who used a similar method to derive their uncertainty estimate of $\pm 1 \mathrm{~dB}$ for $D_{2, \mathrm{~S}}$ and of $\pm 1.5 \mathrm{~dB}$ for $L_{p, \mathrm{~A}, \mathrm{~S}, 4 \mathrm{~m}}$. According to the GUM, these results represent an estimate of the uncertainty attributable to different measurement paths.

A further contribution in this regard is a round-robin test presented by Kelz [8]. Ten laboratories participated in this test, which yielded measurement results of $4.3 \mathrm{~dB} \leq D_{2, \mathrm{~S}} \leq 6.1 \mathrm{~dB}$ and $47.9 \mathrm{~dB} \leq L_{p, \mathrm{~A}, \mathrm{~S}, 4 \mathrm{~m}} \leq 51.1 \mathrm{~dB}$. Since these ranges are the result of all contributions to the uncertainty, they are used in Section 2 to deduce a conservative estimate of the uncertainty caused by different choices of path.

Currently, the lack of a rigorous uncertainty declaration is abridged for example by the work of Lenne et al. [9].

*Corresponding authors: moritz.schneider@dguv.de; jan.selzer@dguv.de 
Although these researchers apply the GUM in the same way as the present paper, they use it mainly to validate their Monte-Carlo simulations in accordance with Supplement 1 of the GUM [11]. They include the uncertainty caused by sound level meters and distance measurements. Since they used the Monte-Carlo simulations to extend the range of the uncertainty for the distance measurements up to $1 \mathrm{~m}$, they were also able to some extent to cover the uncertainty caused by different paths. They stated expanded uncertainties of less than $\pm 1 \mathrm{~dB}$ for $D_{2, \mathrm{~S}}$.

Consideration of (expanded) uncertainties of the order of $1 \mathrm{~dB}$ reported in the available literature shows the ranges of current limit values to be in the same order of magnitude. For example, the German national guideline VDI 2569 [12] supports assignment of open-plan offices to different room acoustics classes from A to C. The classification is carried out using levels of sound propagation [12] for single measurement paths with a step size of $2 \mathrm{~dB}$ for the parameters $D_{2, \mathrm{~S}}$ and $L_{p, \mathrm{~A}, \mathrm{~S}, 4 \mathrm{~m}}$. Furthermore, national standards and guidelines using the single number quantities of ISO 3382-3 are available, for example in France and Finland ([13] and presented in [14]). The French standard focuses on requirements for the $D_{2, \mathrm{~S}}$ and the attenuation $D_{n}$. The Finnish guideline considers the $D_{2, \mathrm{~S}}$ with a step size of $2 \mathrm{~dB}, L_{p, \mathrm{~A}, \mathrm{~S}, 4 \mathrm{~m}}$ with a step size of $3 \mathrm{~dB}$, and also the distraction distance $r_{D}$, based on the Speech Transmission Index (STI), for acoustic classification. Acoustics classes from A to E are available. Draft international standard ISO/DIS 22955 will provide further requirements for different open office types based on the sound pressure level quantities of ISO 3382-3 [15] in the future.

Based on the above, the present work applies the framework of the GUM to $D_{2, \mathrm{~S}}$ and $L_{p, \mathrm{~A}, \mathrm{~S}, 4 \mathrm{~m}}$. It covers the uncertainty components for the measurement equipment, distance measurement and choice of path. This is outlined in the next section together with the background information on the measurements to which we apply the GUM. The resulting equations are stated in Section 3 together with the expanded uncertainties for the measurements. The equations may assist other laboratories in deriving their own uncertainties, since they are easily expanded and do not require additional Monte-Carlo simulations as in [9]. Finally, the results are discussed in Section 4, particular attention being paid to the validity of the approach and possible improvements to it, and to the regulatory requirements mentioned above.

\section{Methods - determining the uncertainty 2.1 GUM - law of uncertainty propagation}

Based on all available information on a measurement, the GUM specifies a procedure for deriving the uncertainty, i.e. a range of values associated with the result of a measurement, that characterizes the dispersion of quantity $Y[10]$. In this case, $Y$ is equal to the parameters $D_{2, \mathrm{~S}}$ and $L_{p, \mathrm{~A}, \mathrm{~S}, 4 \mathrm{~m}}$ of ISO 3382-3. All contributions to the uncertainty are input into this process in the form of variables $X_{i}$ in a function $f$, the mathematical model $Y=f\left(X_{1}, X_{2}, \ldots, X_{M}\right)$.
This model is subject to the law of uncertainty propagation, which comprises a first-order Taylor series expansion for $f$ followed by the formation of expected values. This is dependent upon the $X_{i}$ variables not leading to pronounced changes in $Y$, as the Taylor series expansion is otherwise not valid. These steps yield $\sigma_{Y, c, \text { corr }}$, the combined standard uncertainty of $Y$ :

$$
\begin{aligned}
\sigma_{Y, \mathrm{c}, \mathrm{corr}} & =\sqrt{\sum_{i}\left(\frac{\partial f}{\partial X_{i}}\right)_{\langle\rangle}^{2} \sigma_{Y}(i)^{2}+\sum_{i \neq j}\left(\frac{\partial f}{\partial X_{i}}\right)_{\langle\rangle}\left(\frac{\partial f}{\partial X_{j}}\right)_{\langle\rangle} \sigma_{Y}(i, j)} \\
& =\sqrt{\sum_{i} c_{Y}(i)^{2} \sigma_{Y}(i)^{2}+\sum_{i \neq j} c_{Y}(i) c_{Y}(j) \sigma_{Y}(i, j)}
\end{aligned}
$$

The index "c" refers to combined, the index "corr" to the fact that the $X_{i}$ variables are not required to be independent at this point. In addition, the partial derivatives of $f$ with regard to $X_{i}$ are the sensitivity coefficients $c_{Y}(i)$ :

$$
c_{Y}(i)=\left(\frac{\partial f}{\partial X_{i}}\right)_{\langle\rangle} .
$$

The sensitivity coefficients weight the standard deviations $\sigma_{Y}(i)$ and the covariances $\sigma_{Y}(i, j)$ of the $X_{i}$ variables. The index \langle\rangle indicates that they are evaluated at the expected values. This means that all measurable quantities take the measured values and that the uncertainty components $X_{i}$ are expressed by their expected values $\left\langle X_{i}\right\rangle$.

In addition, statistical independence of the $X_{i}$ variables can be made a requirement. This enables their covariances to be neglected, and Equation (1) becomes,

$$
\sigma_{Y, \mathrm{c}}=\sqrt{\sum_{i} c_{Y}(i)^{2} \sigma_{Y}(i)^{2}} .
$$

In the present work, the combined standard deviation $\sigma_{Y, c}$ describes the dispersion of $Y$. Since the exact standard deviations in Equation (3) are never known, recourse must be made to estimations of the standard deviations $\hat{\sigma}_{Y, \mathrm{c}}, \hat{\sigma}_{Y}(i)$. These estimates are termed uncertainties in the GUM: $u_{c}=\hat{\sigma}_{Y, c}, u_{i}=\hat{\sigma}_{Y}(i)$,

$$
u_{c}=\hat{\sigma}_{Y, \mathrm{c}}=\sqrt{\sum_{i} c_{Y}(i)^{2} \hat{\sigma}_{Y}(i)^{2}} .
$$

Finally, the expanded uncertainty $U=k \cdot u_{c}$ must be constructed, in which $k \approx 2$ is typically selected as the coverage factor $k$, assuming a confidence level close to $95 \%$.

This assumption is motivated by the fact that for many cases the central limit theorem leads to approximately normally distributed values for $Y$. For a normally distributed $Y, k=1.96$ leads to a confidence level of $95 \%$ for $\langle Y\rangle \pm U$, where $\langle Y\rangle$ is the measured value of $Y[16]$.

\subsection{Mathematical model}

The mathematical model $Y=f\left(X_{1}, X_{2}, \ldots, X_{M}\right)$ is created as follows. Firstly, the dependency of $D_{2, \mathrm{~S}}$ and $L_{p, \mathrm{~A}, \mathrm{~S}, 4 \mathrm{~m}}$ upon the measurable quantities $r_{n}$ and $L_{p, \mathrm{Ls}, n, i}$ must be recognized, where $r_{n}$ is the distance of a 
measurement point to the loudspeaker in the measurement path and $L_{p, \mathrm{Ls}, n, i}$ is the sound pressure level of the loudspeaker measured at $r_{n}$ in the octave band $i$. The $L_{p, \mathrm{Ls}, n, i}$ quantities are combined into $L_{p, \mathrm{~A}, \mathrm{~S}, n}$, the A-weighted sound pressure level of speech at the distance $r_{n}$ :

$$
L_{p, \mathrm{~A}, \mathrm{~S}, n}=10 \log _{10}\left(\sum_{i=1}^{7} 10^{0.1\left(L_{p, \mathrm{Ls}, n, j}+C_{i}\right)}\right) .
$$

Here, $C_{i}$ is a sum of constants for the octave band $i$ given by Equation (6). $C_{i}$ comprises the constants $A_{i}$ of the A-weighting and $L_{p, \mathrm{~S}, 1 \mathrm{~m}, i}$, the sound pressure level of omnidirectional speech at a distance of $1 \mathrm{~m}$, both in accordance with Table 1 of ISO 3382-3 [5], and $L_{p, \mathrm{Ls}, 1 \mathrm{~m}, i}$, the sound pressure level at a distance of $1 \mathrm{~m}$ from the acoustic centre of the loudspeaker in a free field, calculated on the basis of the sound power level of the loudspeaker:

$$
C_{i}=A_{i}+L_{p, \mathrm{~S}, 1 \mathrm{~m}, i}-L_{p, \mathrm{Ls}, 1 \mathrm{~m}, i}
$$

The ISO 3382-3 standard assumes a constant decrease of $L_{p, \mathrm{~A}, \mathrm{~S}, n}$ when the distance $r_{n}$ is doubled in the range $2 \mathrm{~m} \leq r_{n} \leq 16 \mathrm{~m}$.

This leads to the following regression model:

$$
L_{p, \mathrm{~A}, \mathrm{~S}, n}=\beta_{0}+\beta_{1} \cdot \log _{2}\left(\frac{r_{n}}{r_{0}}\right)+\epsilon,
$$

where $r_{0}=1 \mathrm{~m}$ is the unit distance and $\epsilon$ is a normally distributed variable with a constant variance. Expected values for the intercept $\hat{\beta}_{0}$ and the slope $\hat{\beta}_{1}$ are formed in ISO 3382-3 by means of a regression analysis [17]. For this purpose, $N$ pairs of the independent variable $\log _{2}\left(\frac{r_{n}}{r_{0}}\right)$ and the dependent variable $L_{p, \mathrm{~A}, \mathrm{~S}, n}$ must be measured. Changing the logarithm base from 2 to 10 and use of the abbreviations $R_{n}=\log _{10}\left(\frac{r_{n}}{r_{0}}\right), L_{n}=L_{p, \mathrm{~A}, \mathrm{~S}, n}$ yields the following definition of $D_{2, \mathrm{~S}}$ :

$$
D_{2, \mathrm{~S}}=-\hat{\beta}_{1}=-\log _{10}(2) \frac{\left[N \sum_{n}^{N} L_{n} R_{n}-\sum_{n}^{N} L_{n} \sum_{n}^{N} R_{n}\right]}{N \sum_{n}^{N} R_{n}^{2}-\left(\sum_{n}^{N} R_{n}\right)^{2}} .
$$

The definition of $L_{p, \mathrm{~A}, \mathrm{~S}, 4 \mathrm{~m}}$ is obtained by setting $r_{n}=4 \mathrm{~m}$ and replacing $\beta_{0,1}$ with their estimates in Equation (7). The estimate $\hat{\beta}_{1}=-D_{2, \mathrm{~S}}$ was introduced in the last equation. The estimate $\hat{\beta}_{0}$ is obtained from the mean values of the dependent $\bar{L}=1 / N \sum L_{n}$ and the independent variables $\bar{R}=1 / N \sum R_{n}[17]$,

$$
\hat{\beta}_{0}=\bar{L}+D_{2, \mathrm{~S}} \cdot \log _{2}(10) \bar{R},
$$

which yields:

$$
\begin{aligned}
L_{p, \mathrm{~A}, \mathrm{~S}, 4 \mathrm{~m}} & =\hat{\beta}_{0}-D_{2, \mathrm{~S}} \cdot \log _{2}\left(\frac{4 \mathrm{~m}}{r_{0}}\right) \\
& =\bar{L}+D_{2, \mathrm{~S}}\left(\log _{2}(10) \bar{R}-2\right) .
\end{aligned}
$$

Equations (8) and (10) show how $D_{2, \mathrm{~S}}$ and $L_{p, \mathrm{~A}, \mathrm{~S}, 4 \mathrm{~m}}$ depend on the measurable quantities $r_{n}$ and $L_{p, \mathrm{Ls}, n, i}$. To complete the mathematical model, the present work now introduces uncertainty components $X_{i}$ for the measurement equipment, the distance measurement and the uncertainty due to different paths. Owing to the lack of further information, we assume an additive influence as follows:

$$
\begin{gathered}
r_{n}=\left\langle r_{n}\right\rangle+X_{\text {dist }, n} \\
L_{p, \mathrm{LS}, n, i}=\left\langle L_{p, \mathrm{LS}, n, i}\right\rangle+X_{\text {equip }, n, i} \\
D_{2, \mathrm{~S}}=\left\langle D_{2, \mathrm{~S}}\right\rangle+X_{\text {path }, D_{2, \mathrm{~S}}} .
\end{gathered}
$$

In this definition, $X_{i}$ are random variables with an expected value of zero: $\left\langle X_{i}\right\rangle=0$. They are added to the measured value of their respective measurable quantities, e.g. $\left\langle r_{n}\right\rangle$ is the measured distance of $r_{n}$ at point $n$.

If Equation (2) is applied to $D_{2, \mathrm{~S}}$ and $L_{p, \mathrm{~A}, \mathrm{~S} .4 \mathrm{~m}}$ as defined in the mathematical model in Equations (8)-(11), the result is the sensitivity coefficients for these parameters. They are presented in Section 3. Note that the additive models of Equation (11) are defined such that their first derivates with respect to $X_{i}$ are always equal to 1 , especially $c_{D_{2, \mathrm{~S}}}\left(X_{\text {path, } D_{2, \mathrm{~S}}}\right)=1$.

\subsection{Estimation of the uncertainty}

The previous subsection described how the sensitivity coefficients $c_{Y}(i)$ can be determined. This subsection is concerned with determination of the uncertainties, i.e. the estimates of the standard deviations $u_{i}=\hat{\sigma}_{i}$. The present work uses the "Type B" approach of the GUM, in which the information on the uncertainty is not derived from repeated dedicated measurements.

Should an estimate for $\hat{\sigma}_{i}$ be found in the literature, the GUM permits its use directly in Equation (4) (Sect. 4.3.4 in [10]). At the same time, it may be assumed that $X_{i}$ exhibits a normal distribution and, based on the principle of maximum entropy $[18,19]$, that $X_{i}$ possesses a uniform distribution, if it modifies the measurand only within a fixed range $\pm b$. The resulting estimate of the standard deviation is then $\hat{\sigma}_{i}=b / \sqrt{3}$. At this point, it must be stressed that the assumption with regard to the form of the distribution does not affect the law of uncertainty propagation. It is however important for a subsequent Monte-Carlo analysis [11].

With this information in mind, the following assumptions can be made:

- Two standards exist containing information on the uncertainty of the measurement of octave bands. One of these is IEC 61672-1 [20], which is used in [9] and is reserved for laboratory measurements. Section C5 of ISO 9612 [21] is therefore used here. This standard, which deals with field measurements, declares an uncertainty of $\hat{\sigma}_{Y}\left(X_{\text {equip }, n, i}\right)=\hat{\sigma}($ equip $)=$ $0.7 \mathrm{~dB}$ for the measured sound pressure level $L_{p, \mathrm{Ls}, n, i}$ for a class 1 sound level meter. This estimate for the uncertainty of measurement equipment is the same for all octave bands $i$, all measurement points $n$, and also for the two quantities $Y=\left\{D_{2, \mathrm{~S}}, L_{p, \mathrm{~A}, \mathrm{~S}, 4 \mathrm{~m}}\right\}$. A normal distribution of $X_{\text {equip }, n, i}$ is assumed in all cases. 
- The accuracy of distance can be measured correctly within a range of $\pm 1 \mathrm{~cm}$ with a tape measure or laser distance meter. A uniform distribution with an uncertainty of $\hat{\sigma}_{Y}\left(X_{\text {dist }, n}\right)=\hat{\sigma}($ dist $)=0.01 \mathrm{~m} / \sqrt{3}$ can therefore be assumed for all measurement points $n$. This estimate is also used for the two quantities $Y=\left\{D_{2, \mathrm{~S}}, L_{p, \mathrm{~A}, \mathrm{~S}, 4 \mathrm{~m}}\right\}$. We assume a uniform distribution of $X_{\text {dist, }, n}$.

- The work of Haapakangas et al. [7] (Sect. 1) states a very large uncertainty with respect to the choice of different measurement paths when compared to the work of Kelz [8]. While the former reports a standard deviation of $1 \mathrm{~dB}$ for $\hat{\sigma}_{D_{2, S}}$, the latter puts forward a range of $1.8 \mathrm{~dB}$ for $D_{2, \mathrm{~S}}$. This range enables the standard deviation of $\hat{\sigma}_{D_{2, S}}\left(X_{\text {path }}\right)=\hat{\sigma}_{D_{2, S}}($ path $)=$ $0.9 \mathrm{~dB} / \sqrt{3} \approx 0.52 \mathrm{~dB}$ to be estimated in the same way as the uncertainty of the distance measurement. This estimate is used here, as it is closer to the estimate of Yadav et al. [6] and refers to ten observed paths rather than the two in [6]. It is a conservative estimate, since the range is based upon all contributions to the uncertainty, and measurement paths have been included that are not strictly consistent with the standard. A uniform distribution is assumed.

- At this point, no further contributions to the uncertainty for $L_{p, \mathrm{~A}, \mathrm{~S}, 4 \mathrm{~m}}$ are assumed beyond those discussed above. They affect the combined uncertainty for $L_{p, \mathrm{~A}, \mathrm{~S}, 4 \mathrm{~m}}$ simply by application of the law of uncertainty propagation to the mathematical model (Eq. (10)).

- Some parts of the random effect from the source (loudspeaker) are addressed in ISO 3741 [22]. In addition, observations during the measurements have shown that $L_{p, \mathrm{~A}, \mathrm{~S}, 4 \mathrm{~m}}$ may be affected by an offset that is constant during one measurement, but can change from one measurement to the other. This might be due to the calibration procedure of the source and should in principle be corrected. However, additional measurements to quantify these effects are beyond the scope of this work. Nevertheless, the impact of the findings in ISO 3741 for one example is discussed below in Section 3.4.2.

The question remains whether or not these contributions to the uncertainty are correlated. It may be safely assumed that the uncertainty of the distance measurement is independent of the other two contributions to the uncertainty. However, the sound pressure level is measured simultaneously in all octave bands at fixed points with a fixed source. For such measurements, the measurement between the octave bands may be correlated at a given measurement point [23]. Since this is caused by the properties of the sound field in the room, correlations between different measurement points are again neglected. Different choices of paths may once again lead to differences between the extent of these correlations. Nevertheless, being aware of these possible correlations, it has to be considered at this point that all contributions to the uncertainty are statistically independent, and Equation (4) is used for the combined uncertainty. In addition, for one measurement, the worst-case situation as described in ISO 12999-1 [24] is assumed, namely a complete positive or negative correlation of the octave-band measurements at each point $n$. In this case, the correlation coefficient is equal to \pm 1 , and the covariance factorises to the two variances: $\sigma_{Y}(i, j)= \pm \sigma_{Y}(i) \sigma_{Y}(j)$. Since the variances estimates are known, the (worst-case) combined uncertainty according to Equation (1) can be calculated in this case.

\subsection{Monte-Carlo simulations}

While the assumption of the form of the distribution does not affect the calculation of $u_{c}$ according to the law of uncertainty propagation, it is important for the numerical calculation of $u_{c}$ by means of Monte-Carlo simulations [11]. With the use of random number generators, the Monte-Carlo simulation calculates a histogram of possible values of $Y$. For each random variable $X_{i}$ in the mathematical model, a number is randomly generated based under the assumed form of the distribution function and the assumed $\hat{\sigma}_{i}$. These numbers enter the Equations (8), (10), (11), and one simulated value for $Y$ is obtained.

The QMSysGUM [25] commercial software package was used in the present work for calculation of $220 \times 10^{3}$ values (trials) for $D_{2, \mathrm{~S}}$ and $L_{p, \mathrm{~A}, \mathrm{~S}, 4 \mathrm{~m}}$. This default value for the Monte-Carlo trials was sufficient for numerical stability of the results to be attained. The normalized histogram of these values can be interpreted as a probability distribution density of $Y$. The $95 \%$ confidence interval is simply the (symmetrical) range around the measured value that covers $95 \%$ of the simulated values. For a normally distributed variable, the skew and the (excess) kurtosis of the histogram are both expected to be close to zero.

Since the Monte-Carlo simulations do not depend on the conditions which are set when formulating Equation (4) (such as Taylor series expansion, independence of $X_{i}$, etc.), Monte-Carlo simulations can be used to validate the results according to the law of uncertainty propagation.

\subsection{Standard error of regression analysis}

If Equation (7), which also yields an estimate of the uncertainty of $D_{2, \mathrm{~S}}$ and $L_{p, \mathrm{~A}, \mathrm{~S}, 4 \mathrm{~m}}$, is correct, the uncertainty due to the distance measurement must be zero or at least much smaller than the variance of $\epsilon$. In addition, this variance must be constant for all $L_{p, \mathrm{~A}, \mathrm{~S}, n}$. If these requirements are met, the sum of the squared residuals can be used to calculate $\hat{\sigma}_{\epsilon}$, an estimate of the variance of $\epsilon$,

$$
\hat{\sigma}_{\epsilon}=\sqrt{\frac{1}{n-2} \sum_{n}\left(L_{p, \mathrm{~A}, \mathrm{~S}, n}-\left(\hat{\beta}_{0}+\hat{\beta}_{1} \log _{2}(10) R_{n}\right)\right)^{2}} .
$$

This estimate comprises all contributions to the uncertainty affecting $L_{p, \mathrm{~A}, \mathrm{~S}, n}$ in a given measurement. In this case, this is restricted to $X_{\text {equip }, n, i}$, as only one path is measured in a given measurement and a negligible distance uncertainty is a requirement.

An estimate can also be formulated for the standard error of $\hat{\beta}_{1}$, namely, $\hat{\sigma}_{1}$ [17]. This is an alternative estimate 
for the combined uncertainty of $D_{2, \mathrm{~S}}$ for one measurement, and since $L_{p, \mathrm{~A}, \mathrm{~S}, n}$ has the same standard error as $\hat{\beta}_{0}$ [17], its standard error $\hat{\sigma}_{0}$ can be used in the same way:

$$
\begin{gathered}
\hat{\sigma}_{1}=\frac{\hat{\sigma}_{\epsilon}^{2}}{\log _{2}(10)^{2} \sum_{n}\left(R_{n}-\bar{R}\right)^{2}} \\
\hat{\sigma}_{0}=\hat{\sigma}_{\epsilon}^{2}\left(\frac{1}{N}+\frac{\bar{R}^{2}}{\log _{2}(10)^{2} \sum_{n}\left(R_{n}-\bar{R}\right)^{2}}\right) .
\end{gathered}
$$

\section{Results}

\subsection{Combined uncertainties}

As outlined in the preceding section, Equation (2) was applied to the mathematical model Equations (8)-(11). This yields the sensitivity coefficients for $D_{2, \mathrm{~S}}$. The $c_{Y}(i)$ must be evaluated at the measured or expected values. Therefore, $r_{n}$ is replaced by the measured value $\left\langle r_{n}\right\rangle$, for example, and $\left\langle X_{i}\right\rangle=0$ by definition. Equation (5) thus becomes,

$$
\begin{aligned}
& \left\langle L_{n}\right\rangle=\left\langle L_{p, \mathrm{~A}, \mathrm{~S}, n}\right\rangle \\
& =10 \log _{10}\left(\sum_{i=1}^{7} 10^{0.1\left(\left\langle L_{p, \mathrm{LS}, n, j}\right\rangle+C_{i}\right)}\right),
\end{aligned}
$$

and for the independent variable in Equation (7) (adjusted to base 10),

$$
\left\langle R_{n}\right\rangle=\log _{10}\left(\frac{\left\langle r_{n}\right\rangle}{r_{0}}\right)
$$

is obtained.

These abbreviations can be used to express the sensitivity coefficients for $D_{2, \mathrm{~S}}$. In the following equation, the index $i$ describes the current octave band and $n$ the current measurement point, while the general summation index is switched to $j$ for the octave bands and to $m$ for the measurement points. This leads to the sensitivity coefficient with respect to the uncertainty in distance measurement:

$$
\begin{aligned}
& c_{D_{2, \mathrm{~S}}}\left(X_{\mathrm{dist}, n}\right)=\left(\frac{\partial D_{2, \mathrm{~S}}}{\partial X_{\mathrm{dist}, n}}\right)_{\langle\rangle}= \\
& -\frac{\log _{10}(2)}{\left\langle r_{n}\right\rangle \cdot \ln (10)} \cdot \frac{1}{\left(N \cdot \sum_{m}^{N}\left\langle R_{m}\right\rangle^{2}-\left(\sum_{m}^{N}\left\langle R_{m}\right\rangle\right)^{2}\right)^{2}} \\
& \cdot\left\{\left[N\left\langle L_{n}\right\rangle-\sum_{m}^{N}\left\langle L_{m}\right\rangle\right] \cdot\left[N \sum_{m}^{N}\left\langle R_{m}\right\rangle^{2}-\left(\sum_{m}^{N}\left\langle R_{m}\right\rangle\right)^{2}\right]\right. \\
& \left.-2\left[N\left\langle R_{n}\right\rangle-\sum_{m}^{N}\left\langle R_{m}\right\rangle\right] \cdot\left[N \sum_{m}^{N}\left\langle L_{m}\right\rangle\left\langle R_{m}\right\rangle-\sum_{m}^{N}\left\langle L_{m}\right\rangle \sum_{m}^{N}\left\langle R_{m}\right\rangle\right]\right\},
\end{aligned}
$$

and the sensitivity coefficient with regard to the uncertainty in measurement equipment is

$$
\begin{aligned}
& c_{D_{2, \mathrm{~S}}}\left(X_{\text {equip }, n, i}\right)=\left(\frac{\partial D_{2, \mathrm{~S}}}{\partial X_{\text {equip }, n, i}}\right)_{\rangle} \\
& =-\frac{\log _{10}(2)}{N \cdot \sum_{m}^{N}\left\langle R_{m}\right\rangle^{2}-\left(\sum_{m}^{N}\left\langle R_{m}\right\rangle\right)^{2}} \cdot \frac{10^{0.1 \cdot\left(\left\langle L_{p, \mathrm{LS}, n, i, i}\right\rangle+C_{i}\right)}}{\sum_{j=1}^{7} 10^{0.1 \cdot\left(\left\langle L_{p, \mathrm{LS}, n, j}\right\rangle+C_{j}\right)}} \\
& \cdot\left\{N \cdot\left\langle R_{n}\right\rangle-\sum_{m}^{N}\left\langle R_{m}\right\rangle\right\} .
\end{aligned}
$$

The last two equations contain only measured values and constants, and by use of the estimates in Section 2.3 and the observation that $c_{D_{2 . S}}\left(X_{\text {path }, D_{2 . S}}\right)=1$, the combined uncertainty is obtained according to Equation (4) for the mathematical model of $D_{2, S}: \hat{\sigma}_{c, D_{2, S}}$. The following equation defines $\hat{\sigma}_{c, D_{2 S}}$ with regard to the three uncertainty components considered in the present work:

$$
\begin{aligned}
\hat{\sigma}_{c, D_{2, \mathrm{~S}}}= & \left(\sum_{n=1}^{N} c_{D_{2, \mathrm{~S}}}\left(X_{\text {dist }, n}\right)^{2} \cdot \hat{\sigma}(\text { dist })^{2}+\hat{\sigma}_{D_{2, \mathrm{~S}}}(\text { path })^{2}\right. \\
& \left.+\sum_{\mathrm{n}=1}^{N} \sum_{\mathrm{i}=1}^{7} c_{D_{2, \mathrm{~S}}}\left(X_{\text {equip }, n, i}\right)^{2} \cdot \hat{\sigma}(\text { equip })^{2}\right)^{0.5} \cdot
\end{aligned}
$$

In the same way, the quantity $L_{p, \mathrm{~A}, \mathrm{~S}, 4 \mathrm{~m}}$ in Equation (10) can now be addressed and the respective sensitivity coefficients calculated, where $\left\langle D_{2, \mathrm{~S}}\right\rangle$ defines the value of $D_{2, \mathrm{~S}}$ when all measured values are inserted, and $\overline{\langle R\rangle}=1 / N \sum\left\langle R_{n}\right\rangle$ is the average of $\left\langle R_{n}\right\rangle$ in Equation (15). The sensitivity coefficient with regard to the uncertainty of the distance measurement is thus,

$$
\begin{gathered}
c_{L_{p, \mathrm{~A}, \mathrm{~S}, \mathrm{~m}}}\left(X_{\text {dist }, n}\right)=\left(\frac{\partial L_{p, \mathrm{~A}, \mathrm{~S}, 4 \mathrm{~m}}}{\partial X_{r_{n}}}\right)_{\langle\rangle}= \\
\left(\frac{\partial D_{2, \mathrm{~S}}}{\partial X_{\text {dist }, n}}\right)_{\langle\rangle} \cdot(\overline{\langle R\rangle}-2)+\left\langle D_{2, \mathrm{~S}}\right\rangle \cdot \frac{\log _{2}(10)}{N \cdot \ln (2)} \cdot \frac{1}{\left\langle r_{n}\right\rangle},
\end{gathered}
$$

and the sensitivity coefficient with regard to the uncertainty of the measurement equipment is,

$$
\begin{aligned}
& c_{L_{p, \mathrm{~A}, \mathrm{~S}, \mathrm{Am}}}\left(X_{\text {equip }, n, i}\right)=\left(\frac{\partial L_{p, \mathrm{~A}, \mathrm{~S}, 4 \mathrm{~m}}}{\partial X_{\text {equip }, n, i}}\right)_{\langle\rangle}=\frac{1}{N} \frac{10^{0.1 \cdot\left(\left\langle L_{p, \mathrm{LS}, n, i}\right\rangle+C_{i}\right)}}{\sum_{j=1}^{7} 10^{0.1 \cdot\left(\left\langle L_{p, \mathrm{Ls}, n, j}\right\rangle+C_{j}\right)}} \\
&+\left(\frac{\partial D_{2, S}}{\partial X_{\text {equip }, n, i}}\right)_{\langle\rangle}\left(\log _{2}(10) \overline{\langle R\rangle}-2\right) . \quad(20)
\end{aligned}
$$

The last two equations depend on Equations (16) and (17) respectively. In addition, the sensitivity coefficient with regard to the uncertainty due to the choice of different paths is,

$$
c_{L_{p, \mathrm{~A}, \mathrm{~S}, 4 \mathrm{~m}}}\left(X_{\mathrm{path}}\right)=\left(\log _{2}(10) \overline{\langle R\rangle}-2\right) .
$$

The combined uncertainty according to Equation (4) for our mathematical model of $L_{p, \mathrm{~A}, \mathrm{~S}, 4 \mathrm{~m}}$ is thus defined by 
the following equation with regard to the three uncertainty components considered in the present work:

$$
\begin{aligned}
\hat{\sigma}_{c, L_{p, \mathrm{~A}, \mathrm{~S}, \mathrm{~m}}} & =\left(\sum_{n=1}^{N} c_{L_{p, \mathrm{~A}, \mathrm{~S}, \mathrm{~m}}}\left(X_{\mathrm{dist}, n}\right)^{2} \cdot \sigma(\text { dist })^{2}\right. \\
& +c_{L_{p, \mathrm{~A}, \mathrm{~S}, 4 \mathrm{~m}}}\left(X_{\mathrm{path}, D_{2, \mathrm{~S}}}\right)^{2} \cdot \hat{\sigma}_{D_{2, \mathrm{~S}}}(\text { path })^{2} \\
& \left.+\sum_{n=1}^{N} \sum_{i=1}^{7} c_{L_{p, \mathrm{~A}, \mathrm{~S}, 4 \mathrm{~m}}}\left(X_{\text {equip }, n, i}\right)^{2} \cdot \hat{\sigma}(\text { equip })^{2}\right)^{0.5} .
\end{aligned}
$$

The Equations (18) and (22) for the sensitivity coefficients are the first result of the present work, as they constitute the first step in calculation of the combined uncertainty for the measurements described in the following Section 3.2.

\subsection{Measurements}

This subsection presents the results of measurements according to ISO 3382-3 that have been conducted as part of noise abatement consultations. Between 2015 and 2019 a total of 17 offices have been examined and some of the results have been presented already in [26]. The number of workstations in the offices varies between 11 and 50 with a median of 30 .

In each office or office zone, two to five measurement paths have been investigated. This gives rise to 44 different measurement paths, each with a different value for $D_{2, \mathrm{~S}}$ and $L_{p, \mathrm{~A}, \mathrm{~S}, 4 \mathrm{~m}}$, that are shown in Figures 1 and 2 . The results of the measurement paths vary within following ranges: $3.0 \mathrm{~dB}<D_{2, \mathrm{~S}}<8.8 \mathrm{~dB}, 43.5 \mathrm{~dB}<L_{p, \mathrm{~A}, \mathrm{~S}, 4 \mathrm{~m}}<54.9 \mathrm{~dB}$ (see Figs. 1 and 2).

The ranges of $D_{2, \mathrm{~S}}$ and $L_{p, \mathrm{~A}, \mathrm{~S}, 4 \mathrm{~m}}$ are similar to those reported in the literature. For example Haapakangas et al. [7] report values from 21 open-plan offices: $3.3 \mathrm{~dB}<D_{2, \mathrm{~S}}<12.1 \mathrm{~dB}, 44.6 \mathrm{~dB}<L_{p, \mathrm{~A}, \mathrm{~S}, 4 \mathrm{~m}}<53.8 \mathrm{~dB}$. Yadav et al. [6] report values from their reliability and repeatability measurements in 27 open-plan offices: $2.6 \mathrm{~dB}<D_{2, \mathrm{~S}}<8.4 \mathrm{~dB}, 46.2 \mathrm{~dB}<L_{p, \mathrm{~A}, \mathrm{~S}, 4 \mathrm{~m}}<56.0 \mathrm{~dB}$.

\subsection{Uncertainty estimates}

Application of the Equations (18) and (22) to the data presented in the previous Section 3.2 yields a range of combined uncertainties: $0.55 \mathrm{~dB} \leq \hat{\sigma}_{c, D_{2 . S}} \leq 0.67 \mathrm{~dB}$ and $0.19 \mathrm{~dB} \leq \hat{\sigma}_{c, L_{p, A, S, 4 \mathrm{~m}}} \leq 0.83 \mathrm{~dB}$, as seen in Figures 3 and 4 .

Based on the dataset of measurements, 11 measurement paths with more than four measurement points per path are available. These dataset is used to recalculate the parameters $\hat{\sigma}_{c, D_{2, S}}$ and $D_{2, \mathrm{~S}}$ with four, five and six consecutive measurement points. For example, when the results for $N=4$ are computed, the last two measurement points are dropped if the measurement was conducted with six measurement points. For $N=5$, the last point was then rejected, and so on. This analysis and the equations above indicate a dependency between the uncertainty and the selected number of measurement points. Increasing the

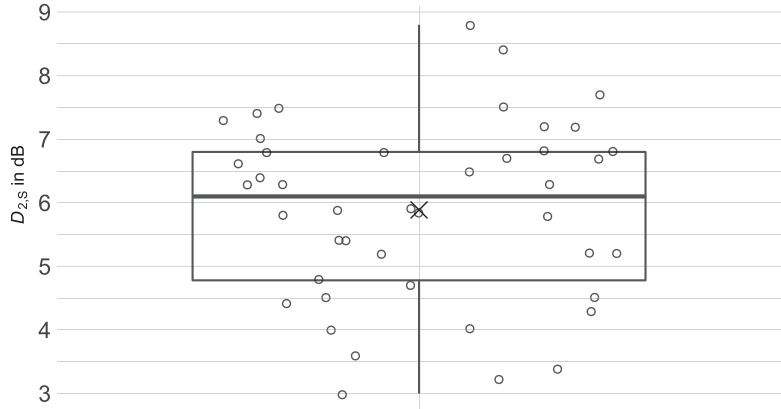

Figure 1. Boxplot representation of all 44 measurement path results for $D_{2, \mathrm{~S}}$. The individual measurement results are indicated as circles, the mean value is represented by $x$.

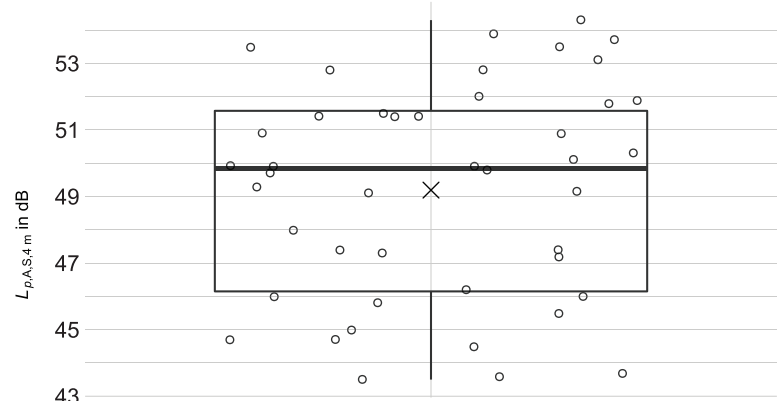

Figure 2. Boxplot representation of all 44 measurement path

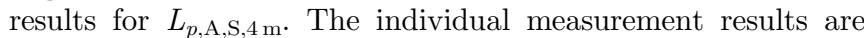
indicated as circles, the mean value is represented by $x$.

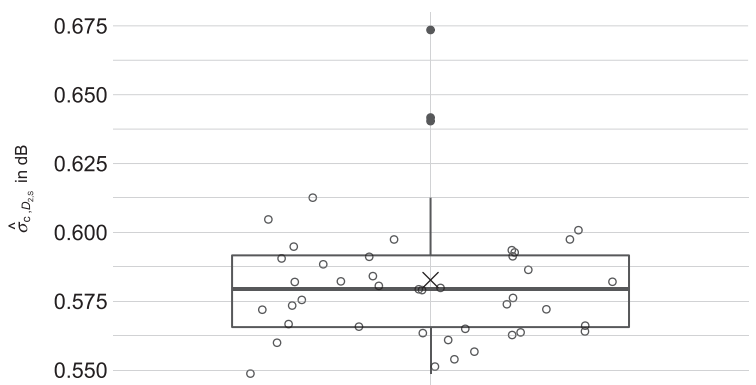

Figure 3. Boxplot of $\hat{\sigma}_{c, D_{2, S}}$ for all 44 measurement paths. The results of the individual calculations are indicated as circles, the mean value is represented by $x$. Three possible outliers are visible.

number of measurement points along a measurement path decreases the overall combined uncertainty $\sigma_{c, D_{2 . S}}$ (see Fig. 5). Omitting measurement points during the measurement or the analysis of a measurement leads to different results in the $D_{2, S}$. The number of measurement points determines the length of the measurement path. Calculating the aforementioned parameter for an increasing measurement path length can increase or decrease $D_{2, \mathrm{~S}}$. Owing to differences in office furniture, a spread of $-1.3 \mathrm{~dB}<\Delta D_{2, \mathrm{~S}}<1.5 \mathrm{~dB}$ occurs in the measurement 


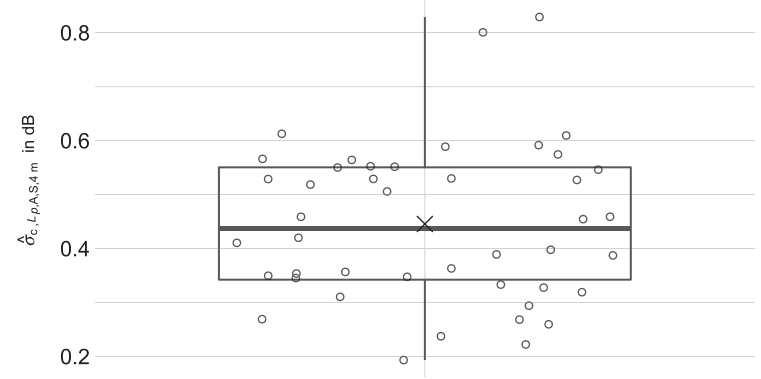

Figure 4. Boxplot of $\hat{\sigma}_{c, L_{p, A, S 4 \mathrm{~m}}}$ for all 44 measurement paths. The results for the individual calculations are indicated as circles, the mean value is represented by $x$.

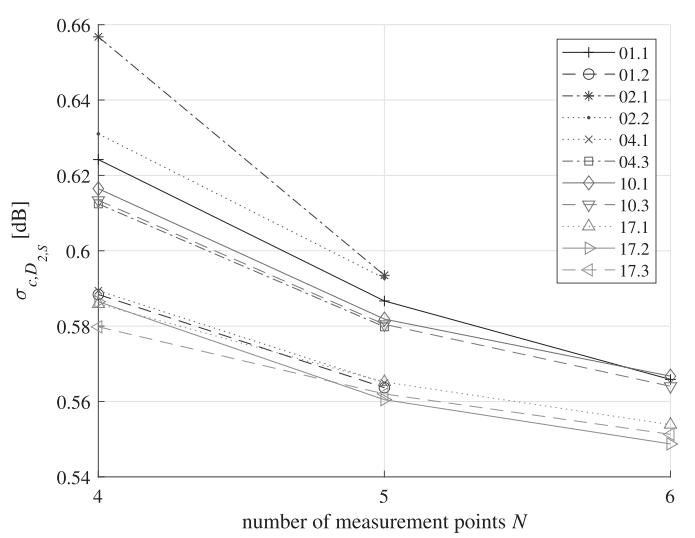

Figure 5. Decrease in combined uncertainty $\hat{\sigma}_{c, D_{2, S}}$ with increasing number of measurement points $N$ along the same measurement paths. Legend shows the number of the office followed by the number of the path.

results across the same measurement paths with changing numbers of measurement points $N$.

\subsection{Detailed results of one example}

In this subsection, detailed results from one measurement consisting of $N=6$ measurement points are presented. The measured parameters are $D_{2, \mathrm{~S}}=8.8 \mathrm{~dB}$ and $L_{p, \mathrm{~A}, \mathrm{~S}, 4 \mathrm{~m}}=49.2 \mathrm{~dB}$. The combined uncertainties of these parameters due to the law of uncertainty propagation are $\hat{\sigma}_{c, D_{2, S}}=0.567 \mathrm{~dB}$ and $\hat{\sigma}_{c, L_{p, A, S, 4 \mathrm{~m}}}=0.552 \mathrm{~dB}$. With a coverage factor of $k=2$, this amounts for both parameters to an uncertainty range of $\pm U= \pm 1.1 \mathrm{~dB}$, which can be attributed to the measurement result with a confidence of $95 \%$.

\subsubsection{Relevance of contributions to the uncertainty}

Since all quantities in Equations (18) and (22) are positive, the relevance of the different contributions to the uncertainty can easily be expressed as the percentage that they add to the combined uncertainty. Firstly, it must be noted that the different contributions to the uncertainty have approximately the same percentages with regard to the combined uncertainties of $D_{2, \mathrm{~S}}$ and $L_{p, \mathrm{~A}, \mathrm{~S}, 4 \mathrm{~m}}$. The percentages for $\hat{\sigma}_{c, D_{2, S}}$ are therefore discussed below and the respective numbers for $L_{p, \mathrm{~A}, \mathrm{~S}, 4 \mathrm{~m}}$ indicated in brackets.

The most important contribution stems from the uncertainty caused by the choice between different paths. This alone accounts for $85 \%$ (75\%) of the combined uncertainty. Without this effect, the combined uncertainty for this measurement would be $0.226 \mathrm{~dB}(0.277 \mathrm{~dB})$. This effect is followed by the uncertainty caused by the equipment in the sound pressure measurement for the octave band around $500 \mathrm{~Hz}$. This accounts for $13 \%(20 \%)$ of the combined uncertainty, and the same effect for the octave band at $1000 \mathrm{~Hz}$ accounts for 3\% (5\%). A closer look at these two octave bands shows that the contributions for measurement points $n=\{1,2,5,6\}$ are more important for the combined uncertainty than those in the middle of the path $n=\{3,4\}$. The sum of all other contributions (other octave bands, the distance measurement) amounts to less than $1 \%$ of the combined uncertainties.

\subsubsection{Uncertainty of the sound source}

This work assumes in Equation (6) that $L_{p, \mathrm{Ls}, 1 \mathrm{~m}, i}$, the sound pressure level at a distance of $1 \mathrm{~m}$, is a constant, although it is a measurable quantity and thus affected by uncertainty. For example, the International Standard ISO 3741 [22] is mainly concerned with the uncertainty attributed to the determination of the sound power level of a given source in a reverberation room. In addition, it describes that the sound pressure of a source is affected by mounting and operating conditions during field measurements. The former effect is attributed to an uncertainty $\hat{\sigma}_{\mathrm{R} 0}$, the latter to $\hat{\sigma}_{\text {omc }}$. This leads to a combined uncertainty of $\hat{\sigma}_{\text {tot }}=$ $\sqrt{\hat{\sigma}_{\mathrm{R} 0}^{2}+\hat{\sigma}_{\mathrm{omc}}^{2}}$. The influence of the instability of the sound power level of the source under investigation $\hat{\sigma}_{\text {omc }}^{2}$ can be measured as the uncertainty due to repeatability in a round-robin test. It is mainly affected by the stability of the acoustic condition in the given room and is likely to outnumber $\hat{\sigma}_{\mathrm{R} 0}$ [22]. The informative Annex G of ISO 3741 gives a guidance value of $\hat{\sigma}_{\text {tot }}=\sqrt{0.5^{2}+0.7^{2}} \mathrm{~dB} \approx$ $0.9 \mathrm{~dB}$, assuming a high-precision measurement $\hat{\sigma}_{\mathrm{R} 0}=$ $0.5 \mathrm{~dB}$ and stable operating conditions $\hat{\sigma}_{\mathrm{omc}}=0.7 \mathrm{~dB}$.

With this information, the mathematical model in Equation (6) can be adapted to,

$$
C^{\prime}{ }_{i}=A_{i}+L_{p, \mathrm{~S}, 1 \mathrm{~m}, i}-\left(L_{p, \mathrm{Ls}, 1 \mathrm{~m}, i}+X_{\text {source }}\right),
$$

where $X_{\text {source }}$ is a normally distributed random number with expected value zero and variance $\hat{\sigma}_{\text {tot }}^{2}=0.9 \mathrm{~dB}$. As a result, the combined uncertainty $\hat{\sigma}_{c, D_{2, S}}$ increases from $0.57 \mathrm{~dB}$ to $0.64 \mathrm{~dB}$. It is clear that this effect is not negligible. However, since a better description of operating conditions of sound sources in open-plan offices requires additional measurements, it is beyond the scope of this work. The same holds true for a constant offset $\Delta$ for $L_{p, \mathrm{Ls}, 1 \mathrm{~m}, i}$, that can be corrected by subtracting $\Delta$ from $L_{p, \mathrm{Ls}, 1 \mathrm{~m}, i}$ in Equation (23). 


\subsubsection{Possible correlations}

As outlined in Section 2.3, a complete positive or negative correlation at the measurement points for the $X_{\text {equip }, n, i}$ at a given measurement point $n$ was assumed. This leads to the addition of the following term in the brackets of Equations (18) and (22),

$$
\begin{gathered}
\pm \sum_{n=1}^{N} \sum_{i \neq j} c_{L_{p, A, S, 4 \mathrm{~m}}}\left(X_{\text {equip }, n, i}\right) c_{L_{p, A, S, 4 \mathrm{~m}}}\left(X_{\text {equip }, n, j}\right) \cdot \hat{\sigma}(\text { equip })^{2} \\
\approx \pm 0.05 \mathrm{~dB}
\end{gathered}
$$

where the fact that the estimates for the standard deviations $\hat{\sigma}$ (equip) are identical in all octave bands has been used. As a result, the combined correlated uncertainty for $D_{2, \mathrm{~S}}$ is $0.521 \mathrm{~dB} \leq \hat{\sigma}_{\mathrm{c}, \text { corr }, D_{2, \mathrm{~S}}} \leq 0.601 \mathrm{~dB}$. For $L_{p, \mathrm{~A}, \mathrm{~S}, 4 \mathrm{~m}}$ this amounts to $0.505 \mathrm{~dB} \leq \hat{\sigma}_{\mathrm{c}, \text { corr }, D_{2 . S}} \leq 0.596 \mathrm{~dB}$. For both parameters, the correlation effect would change the combined uncertainty by less than $10 \%$ were it to exist.

\subsubsection{Monte-Carlo simulation}

In the next step, a Monte-Carlo simulation was used to determine the uncertainty. To this end, simulated values of $D_{2, \mathrm{~S}}$ and $L_{p, \mathrm{~A}, \mathrm{~S}, 4 \mathrm{~m}}$ based on the mathematical model of Section 2 and the distributions assumed there. As a result, $95 \%$ of the simulated values were in a range of $\pm U=$ $\pm 1.0 \mathrm{~dB}$ around the measured values of $D_{2, \mathrm{~S}}$ and $L_{p, \mathrm{~A}, \mathrm{~S}, 4 \mathrm{~m}}$. The histograms of $D_{2, \mathrm{~S}}$ and $L_{p, \mathrm{~A}, \mathrm{~S}, 4 \mathrm{~m}}$ deviate from a normal distribution. The estimate for the skew is 0.002 , that for the (excess) kurtosis -0.854 . We thus observe a platykurtic distribution with large shoulders and smaller tails.

To investigate this deviation, the Monte-Carlo simulation was recalculated with three changes. Firstly, a normal distribution for $X_{\text {path, } D_{2 . S}}$ was assumed. Secondly, $X_{\text {path, } D_{2 . S}}$ was neglected. Finally, the estimated uncertainty for $X_{\text {path }, D_{2, S}}$ to $\hat{\sigma}_{D_{2, S}}($ path $)=0.4 \mathrm{~dB} / \sqrt{3}$ was reduced. In all three cases, good agreement is achieved between the histograms and the results of Equation (4) with an assumed normal distribution (almost identical expanded uncertainty ranges, kurtosis close to zero).

\subsubsection{Standard error of regression analysis}

To confirm the requirement of constant variance of $\varepsilon$ we have calculated the standard uncertainties of $L_{p, \mathrm{~A}, \mathrm{~S}, n}$ according to the framework outlined in Section 2 . We omit the details for the sake of brevity. The standard uncertainties of $L_{p, \mathrm{~A}, \mathrm{~S}, n}$ cover a comparatively close range between $0.44 \mathrm{~dB}$ and $0.48 \mathrm{~dB}$, and since the uncertainty due to the distance measurement is negligible, the requirements for calculation of the standard error of the regression coefficients according to Equations (13) are met. These amount to $\hat{\sigma}_{1}=0.52 \mathrm{~dB}$ for $D_{2, \mathrm{~S}}$ and $\hat{\sigma}_{0}=1.58 \mathrm{~dB}$ for $L_{p, \mathrm{~A}, \mathrm{~S}, 4 \mathrm{~m}}$. These estimates must be compared with the estimates without path uncertainty, i.e. $0.226 \mathrm{~dB}$ and $0.277 \mathrm{~dB}$. The higher values for the standard errors from the regression analysis are due to the fact that the residuals alone cause a variance of $\hat{\sigma}_{\epsilon} \approx 1.05 \mathrm{~dB}$.

\section{Discussion}

Based on the justifications for the approach given above, it has been a conscious choice to present the results using the law of uncertainty propagation (Eq. (4)). Lenne et al. [9] have already shown that recourse need not be made to Monte-Carlo simulations unless investigation of extreme cases is desired, such as distance measurements varying by more than $0.5 \mathrm{~m}$. In addition, even this approach does not fully cover the uncertainty due to different paths, which is caused not by a (large) uncertainty in the distance measurement, but by completely different outlines of measurement paths in a given open-plan office. This uncertainty is more appropriately expressed by a separate term in the mathematical model, as in Equation (11).

However, the inclusion of this parameter has caused a deviation from the normal distribution in the Monte-Carlo simulation presented here. In principle, this deviation can be ignored, since the normality assumption leads only to a larger and therefore more conservative uncertainty range. On the other hand, the additional Monte-Carlo simulations show that if the available information on $X_{\mathrm{path}, D_{2, S}}$ can be improved, good agreement with Equation (4) and normally distributed parameters $Y$ is attained. Since only a range of $D_{2, \mathrm{~S}}$ values has been reported in [8], one has no choice but to assume a uniform distribution due to the principle of maximum entropy. If a larger sample and a better analysis (i.e. a $\hat{\sigma}$ instead of a range) were available, a normal distribution could be assumed. In addition, the reported range is known to be caused by all contributions to the uncertainty. The estimate for the path uncertainty is therefore too large. As shown, for a normally distributed $X_{\text {path, } D_{2 . S}}$ or a smaller uncertainty estimate for $X_{\text {path, } D_{2 . S}}$ the results of Equation (4) and the normality assumption for $Y$ would coincide again with Monte-Carlo simulations. This deviation does not therefore impair the use of Equation (4).

On the contrary, the Monte-Carlo simulations show the best agreement with the analytical results of Equation (4) for the other contributions to the uncertainty. This supports several assumptions. Firstly, the assumed uncertainties are still sufficiently small to justify the first-order Taylor expansion. Secondly, the distribution assumptions for the influences $X_{i}$ are justified. Finally, the choice of $k=2 \approx 1.96$ is justified, since the numerical distribution of $Y=\left\{D_{2, \mathrm{~S}}, L_{p, \mathrm{~A}, \mathrm{~S}, 4 \mathrm{~m}}\right\}$ is very close to the normal distribution. There is therefore no need to resort to numerical simulations, since Equation (4) offers a reliable approach. Nor is this conjecture changed by possible correlation between octave band measurements: even were this effect to be present, it would not amount even in the worst case to more than $10 \%$ of the combined uncertainty in the analysed measurement.

For these reasons, the approach of Equation (4) has been considered the best means of expressing uncertainty for $D_{2, \mathrm{~S}}$ and $L_{p, \mathrm{~A}, \mathrm{~S}, 4 \mathrm{~m}}$. The alternative approach of standard errors in Equation (13) is restricted to very limited cases and is based solely on information obtained from small samples (in our case $N=6$ ). The latter is one reason for the deviation of the standard errors from the respective 
uncertainties determined in the present work. The other reason is that the present work has also neglected certain contributions to the uncertainty, such as repeatability and the sound pressure level of the loudspeaker. These are discussed below.

Conversely, Monte-Carlo simulations are needed only in extreme cases, if the requirements of Equation (4) are no longer met [9]. In addition, the approach of Equation (4) is flexible and permits a transparent discussion of the available information. The detailed presentation of the results for the sensitivity coefficients in the previous section should therefore encourage further laboratories to verify their own uncertainty calculations. In addition, the mathematical model presented here is easily adapted to cases in which more or other information is available:

- Unfortunately, only a worst-case estimate for the largest contribution, namely the path uncertainty, is available. This problem could be addressed in a future round-robin test (see Sect. 3.4.3).

- The ranges reported in the literature for $L_{p, \mathrm{~A}, \mathrm{~S}, 4 \mathrm{~m}}$ [6-8] and the estimate from the standard error in the present work indicate that we do not have access to a contribution to the uncertainty for this parameter. This additional uncertainty could be caused by an uncertainty due to a constant offset $\Delta$ of the sound pressure level of the source (loudspeaker) which

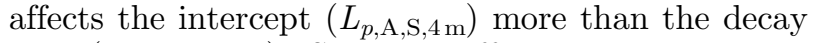
rate (slope, $D_{2, \mathrm{~S}}$ ). Such an offset together with a non-negligible uncertainty of $L_{p, \mathrm{Ls}, 1 \mathrm{~m}, i}$ has to be addressed in future measurements (see Sect. 3.4.2).

- A round-robin test could also address the question of possible correlations in the measurement of the sound pressure level in the octave bands.

- It is obvious from the mathematical model that the uncertainty with respect to repeatability is not yet incorporated. However, a variable $X_{\text {rep }}$ can easily be added in Equation (11) with an uncertainty estimate that might also be determined in a round-robin test (repeatability or precision in [27]). This could also help to explain the difference in the spread of $L_{p, \mathrm{~A}, \mathrm{~S}, 4 \mathrm{~m}}$ between the literature and the present work.

- Besides the uncertainty due to repeatability, a roundrobin test usually summarizes all other contributions to the uncertainty in a single uncertainty estimate, the trueness or reproducibility due to the differences between participating laboratories. However, a large part of this estimate is already covered here.

Based on these points, we expect a better description of the path uncertainty to yield a decrease in the combined uncertainty calculated in the present work. In addition, the results of Figure 5 indicate that the uncertainty decreases with an increasing number of measurement points. It therefore seems reasonable to use more than the minimum number of measurement points wherever this is possible. At the same time, an increase in the uncertainty due to missing terms as discussed above is expected. Overall, the order of magnitude of the expanded uncertainty of $\pm 1 \mathrm{~dB}$ appears realistic, not least in the light of the cited literature.
This raises the question how $D_{2, \mathrm{~S}}$ and $L_{p, \mathrm{~A}, \mathrm{~S}, 4 \mathrm{~m}}$ can be assessed if they are connected with this uncertainty range.

The knowledge gained by the present work indicates that a spread of $2 \mathrm{~dB}$ for one of the two parameters is too small for classification of the acoustic quality of an office, since with the expanded uncertainty $U$ determined for both parameters, $D_{2, \mathrm{~S}}$ and $L_{p, \mathrm{~A}, \mathrm{~S}, 4 \mathrm{~m}}$, a value range of $2 \mathrm{~dB}$ is already covered. This is too large to neglect. A classification scheme with a step size of $3 \mathrm{~dB}$ is more suitable, as presented for the $L_{p, \mathrm{~A}, \mathrm{~S}, 4 \mathrm{~m}}$ in [14]. Where requirements for open offices are defined based on the sound pressure level single number quantities of ISO 3382-3, the findings presented in the present work should also be taken into account.

\section{Conclusion and outlook}

With this work, a contribution to the calculation of expanded uncertainties for the measurement of sound pressure level parameters in accordance with ISO 3382-3 is presented which as yet has been missing in the peer-reviewed literature. The declaration of expanded uncertainties is necessary for the comparison of measurement results between laboratories, assessment of noise reduction measures and classification of offices by means of limit values.

The calculation rests upon the law of uncertainty propagation for uncorrelated influences and this choice has been justified. The equations presented here are intended to facilitate its use by other laboratories. A revision of limit values should reflect the published information on expanded uncertainties, which is expected to be of the order of $\pm 1 \mathrm{~dB}$.

Uncertainty calculations can never be exhaustive. The contribution made by this document will therefore, we hope, be improved by round-robin tests in the future.

\section{Conflict of interest}

Author declared no conflict of interests.

\section{Acknowledgments}

We sincerely thank Ingo Albrecht for his valuable support in performing the measurements.

\section{References}

1. J. Kim, R. de Dear: Workspace satisfaction: The privacycommunication trade-off in open-plan offices. Journal of Environmental Psychology 36 (2013) 18-26.

2. C. Bodin Danielsson, L. Bodin: Difference in satisfaction among employees in different office types. Journal of Architectural and Planning Research 26, 3 (2009) 241-256.

3. C. Bodin Danielsson, H. Singh Chungkham, C. Wulff, H. Westerlund: Office design's impact on sick leave rates. Ergonomics 57, 2 (2014) 139-147.

4. S.J. Schlittmeier, A. Liebl: The effects of intelligible irrelevant background speech in offices - cognitive disturbance, annoyance, and solutions. Facilities 33, 1/2 (2015) 61-75. 
5. International Organization for Standardization: Acoustics Measurement of room acoustic parameters - Part 3: Open plan offices (ISO Standard No. 3382-3). 2012.

6. M. Yadav, D. Cabrera, J. Love, J. Kim, J. Holmes, H. Caldwell, R. de Dear: Reliability and repeatability of ISO 3382-3 metrics based on repeated acoustic measurements in open-plan offices. Applied Acoustics 150 (2019) 138-146.

7. A. Haapakangas, V. Hongisto, M. Eerola, T. Kuusisto: Distraction distance and perceived disturbance by noise - An analysis of 21 open-plan offices. Journal of the Acoustical Society of America 141, 1 (2017) 127-136.

8. P. Kelz: Ringversuch zur Raumakustischen Messung von Großraumbüros gemäß DIN EN ISO 3382-3, in 44th German Annual Conference on Acoustics, DAGA München. 2018, pp. 402-404.

9. L. Lenne, P. Chevret, E. Parizet: Evaluation of Measurement Uncertainties of the $\mathrm{D}_{2 \mathrm{~S}}$ in Open-Plan Offices, in 23rd International Congress on Acoustics (ICA), Aachen, 9-13 of September. 2019, pp. 2423-2428.

10. Joint Committee for Guides in Metrology: Evaluation of measurement data - Guide to the expression of uncertainty in measurement (JCGM Standard No. 100). 2008.

11. Joint Committee for Guides in Metrology: Evaluation of measurement data Supplement 1 to the "Guide to the expression of uncertainty in measurement" - Propagation of distributions using a Monte Carlo method (JCGM Standard No. 101). 2008.

12. The Association of German Engineers [Verein Deutscher Ingenieure - VDI]: Sound protection and acoustical design in offices (VDI National Standard No. 2569). 2019.

13. Association Francaise de Normalisation: Acoustics - Acoustic performance of open-plan offices (Norme francaise No. NF S 31-199). 2016.

14. V. Hongisto, J. Keränen: Open-plan offices - New Finnish room acoustic regulations, in 11th European Congress and Exposition on Noise Control Engineering (Euronoise), Crete, 27-31 of May. 2018, pp. 1147-1153.
15. International Organization for Standardization: Acoustics Acoustic quality of open office spaces (ISO Draft International Standard No. 22955). 2020.

16. S. Bell: Measurement Good Practice Guide No. 11. A Beginner's Guide to Uncertainty of Measurement. National Physical Laboratory, 1999.

17. N.R. Draper, H. Smith: Applied Regression Analysis. Wiley, 1998.

18. E.T. Jaynes: Information theory and statistical mechanics. Physical Review 106, 4 (1957) 620-630.

19. E.T. Jaynes: Information theory and statistical mechanics II. Physical Review 108, 2 (1957) 171-190.

20. International Electrotechnical Commission: Electroacoustics Sound level meters - Part 1: Specifications (IEC Standard No. 61672-1). 2002.

21. International Organization for Standardization: Acoustics Determination of occupational noise exposure - Engineering method (ISO Standard No. 9612). 2009.

22. International Organization for Standardization: Acoustics Determination of sound power levels and sound energy levels of noise sources using sound pressure - Precision methods for reverberation test rooms (ISO Standard No. 3741). 2010.

23. V. Wittstock: On the uncertainty of single-number quantities for rating airborne sound insulation. Acta Acustica United with Acustica. 93, 3 (2007) 375-386.

24. International Organization for Standardization: Acoustics Determination and application of measurement uncertainties in building acoustics - Part 1: Sound insulation (ISO Standard No. 12999-1). 2014.

25. Qualisyst Ltd: QMSys GUM Professional, Server 4.10, 2018.

26. J. Selzer, F. Schelle: Practical aspects of measuring acoustics in German open plan offices, in 11th European Congress and Exposition on Noise Control Engineering (Euronoise), Crete, 27-31 of May. 2018, pp. 1919-1924.

27. International Organization for Standardization: Accuracy (trueness and precision) of measurement methods and results - Part 2: Basic method for the determination of repeatability and reproducibility of a standard measurement method (ISO Standard No. 5725-2). 2019.

Cite this article as: Schneider M. Selzer J. Rissler J. Wolff A. \& Schelle F, et al. 2021. Uncertainties of ISO 3382-3 sound pressure level quantities. Acta Acustica, 5, 27. 